\title{
NÓDULO DA IRMÃ MARIA JOSÉ - UM SINAL SEMIOLÓGICO DE ALERTA: RELATO DE CASO
}

\author{
Sister Mary-Joseph nodule - a semiological sign of alert: case report abstract
}

\section{Jéssica Lima Carvalhido Antonio ${ }^{1}$ Rafael Ruggeri Magalhães ${ }^{1}$ Klícia Magalhães Pereira ${ }^{1}$ Ana Beatriz Esteves Batista ${ }^{2}$ Joaquim Ferreira de Paula ${ }^{3}$ Carlos Augusto Marques Batista ${ }^{3}$}

${ }^{1}$ Centro Universitário de Valença (UNIFAA) Valença $(R J)$

${ }^{2}$ Médica - Rio de Janeiro $(R J)$

${ }^{3}$ Hospital Escola de Valença - Valença (RJ)

\section{Autor correspondente:}

Carlos Augusto Marques Batista

E-mail: carlos.batista@faa.edu.br

\section{Como citar este artigo:}

ANTONIO, J.L.C.; MAGALHÃES, R.R.; PEREIRA, K.M.; BATISTA, A.B.E.; PAULA, J. F.; BATISTA C.A.M. Nódulo da Irmã Maria José - um sinal semiológico de alerta: relato de caso. Revista Saber Digital, v. 14, n. 1, 39-50, 2021.

Data de Submissão: 29/03/2021 Data de publicação: 03/05/2021

\section{RESUMO}

Introdução: O Sinal da Irmã Maria José foi descrito por Hamilton Bailey em 1949, em homenagem à assistente cirúrgica do Dr. William Mayo. É um nódulo endurecido e de crescimento rápido, fissurado ou ulcerado e com aparecimento na região umbilical por via linfática, hematogênica ou contiguidade. O estômago e cólon no homem e ovários na mulher são os locais primários mais comuns. Objetivo: Apresentar um relato de caso sobre o sinal semiológico de Irmã Maria José e sua relação com neoplasias malignas. Relato de caso: Mulher de 71 anos, encaminhada pela Unidade Básica de Saúde (UBS) para procurar um cirurgião geral, com diagnóstico de "hérnia umbilical provavelmente encarcerada". Apresentava tumoração ulcerada em cicatriz umbilical. Tomografia abdominal mostrou múltiplas formações expansivas com calcificações grosseiras, de limites mal definidos em cavidade pélvica. Biópsia da lesão mostrou adenocarcinoma com padrão micropapilar erodindo a pele. O diagnóstico definitivo foi de câncer ovariano avançado, sendo optado apenas por tratamento clínico oncológico. Conclusão: O sinal da Irmã Maria José pode ser a primeira manifestação de uma neoplasia e seu reconhecimento é importante por parte do médico, para que se possa iniciar uma investigação diagnóstica e propor um tratamento adequado, muito embora caracterize um estádio avançado da neoplasia.

Palavras-chave: nódulo da Irmã Maria José; neoplasia de ovário; metástase umbilical.

\section{ABSTRACT}

Introduction: The Sister Mary Joseph Sign was described by Hamilton Bailey in 1949, in honor of Dr. William Mayo's surgical assistant. It is a hardened and fast-growing nodule, fissured or ulcerated and appearing in the umbilical region via lymphatic, hematogenous or contiguous routes. The stomach and colon in men and ovaries in women are the most common primary sites. Objective: To present a case report on the semiological sign of Sister Maria José and its relationship with malignant neoplasms. Case report: A 71year-old woman, referred by Basic Health Unit (UBS) to look for a general surgeon, diagnosed with "probably imprisoned umbilical hernia". She had an ulcerated tumor on the umbilical scar. Abdominal tomography showed multiple expansive formations with gross calcifications, with ill-defined limits in the pelvic cavity. Biopsy of the lesion showed adenocarcinoma with a micropapillary pattern eroding the skin. The definitive diagnosis was advanced ovarian cancer, and only clinical cancer treatment was chosen. Conclusion: The sign of Sister Mary Joseph may be the first manifestation of a neoplasm and its recognition is important on the part of the doctor, so that a diagnostic investigation can be initiated and an appropriate treatment can be proposed, even though it characterizes an advanced stage of the neoplasm.

Keywords: sister Mary Joseph Nodule; ovarian neoplasm; umbilical metastasis. 
Nódulo da Irmã Maria José - um sinal semiológico de alerta: relato de caso Antônio JLC, Magalhães RR, Pereira KM, Batista ABE, Paula JF, Batista CAM

\section{INTRODUÇÃO}

O epônimo "nódulo da Irmã Maria José - NIMJ" ("Sister Mary-Joseph Nodule") foi descrito em publicação por Hamilton Bailey em 1949, em homenagem à assistente cirúrgica do Dr. William Mayo (Mary Joseph Dempsey) no St. Mary's Hospital (atual Mayo Clinic), que observou pela primeira vez a relação dos nódulos metastáticos umbilicais com malignidade intra-abdominal inoperável avançada (ESPERTO; CANHA, 2012; FOGAÇA et al., 2003; GATTU et al, 2020; GIBSON et al., 2018; MURINELLO et al, 2010; O'CONNOR-BYRNE et al., 2020; PANARO et al., 2005; PEDRO et al., 2017; PEREIRA et al., 2011; POPARIC et al., 2017; RENDÓN; RIVEROS; HOYOS, 2013; ZHANG; SANKEY, 2015). Alguns autores afirmam que o NIMJ é mais comum no sexo feminino (GIBSON et al., 2018; NIKOLAOU et al., 2013).

$O$ achado é frequentemente um nódulo endurecido e de crescimento rápido, ulcerado e de coloração variada (FOGAÇA et al., 2003; GIBSON et al., 2018; HUSEIN-ELAHMED et al., 2010; MURINELLO et al, 2010; NIKOLAOU et al., 2013; O'CONNOR-BYRNE et al., 2020; PEDRO et al., 2017; PEREIRA et al., 2011; POPARIC et al., 2017; RENDÓN; RIVEROS; HOYOS, 2013; VEKARIYA et al., 2020; ZHANG; SANKEY, 2015).

Sua confirmação é feita por biópsia incisional ou excisional ou por punção aspirativa (FOGAÇA et al., 2003; GIBSON et al., 2018; HUSEIN-ELAHMED et al., 2010; NIKOLAOU et al., 2013; PANARO et al., 2005; PEDRO et al., 2017; PEREIRA et al., 2011; POPARIC et al., 2017; RENDÓN; RIVEROS; HOYOS, 2013; VEKARIYA et al., 2020; ZHANG; SANKEY, 2015), sendo o tipo histológico mais encontrado o adenocarcinoma (GIBSON et al., 2018; PANARO et al., 2005; PEDRO et al., 2017; PEREIRA et al., 2011; POPARIC et al., 2017).

A imuno-histoquímica é realizada para definição do sítio primário da neoplasia (FOGAÇA et al., 2003; PEREIRA et al., 2011; POPARIC et al., 2017; VEKARIYA et al., 2020). 
As neoplasias gastrointestinais e as neoplasias ginecológicas são os locais primários mais comuns (ESPERTO; CANHA, 2012; FOGAÇA et al., 2003; GIBSON et al., 2018; NIKOLAOU et al., 2013; O'CONNOR-BYRNE et al., 2020; MURINELLO et al, 2010; PANARO et al., 2005; PEDRO et al., 2017; PEREIRA et al., 2011; POPARIC et al., 2017; RENDÓN; RIVEROS; HOYOS, 2013; VEKARIYA et al., 2020; ZHANG; SANKEY, 2015).

Seu aparecimento pode ser por via linfática, hematogênica ou contiguidade (FOGAÇA et al., 2003; MURINELLO et al, 2010; PANARO et al., 2005; PEDRO et al., 2017; PEREIRA et al., 2011; POPARIC et al., 2017; RENDÓN; RIVEROS; HOYOS, 2013; VEKARIYA et al., 2020).

O tratamento varia conforme o tipo de lesão primária e o estado clínico do paciente (FOGAÇA et al., 2003; GIBSON et al., 2018; HUSEIN-ELAHMED et al., 2010; MURINELLO et al, 2010; NIKOLAOU et al., 2013; PEDRO et al., 2017; POPARIC et al., 2017; RENDÓN; RIVEROS; HOYOS, 2013).

O NIMJ indica doença avançada e mau prognóstico (ESPERTO; CANHA, 2012; FOGAÇA et al., 2003; GIBSON et al., 2018, MURINELLO et al, 2010; NIKOLAOU et al., 2013; PANARO et al., 2005; PEDRO et al., 2017; PEREIRA et al., 2011; POPARIC et al., 2017; RENDÓN; RIVEROS; HOYOS, 2013; VEKARIYA et al., 2020; ZHANG; SANKEY, 2015).

O presente trabalho tem como objetivo relatar um caso de neoplasia ovariana diagnosticada com a apresentação do Sinal da Irmã Maria José e mostrar a importância do seu conhecimento e a valorização de um exame físico completo e detalhado. O trabalhou obteve aprovação do Comitê de Ética em Pesquisa do Curso de Medicina da UNIFAA.

\section{RELATO DE CASO}

Mulher de 71 anos de idade, foi encaminhada pela UBS para procurar um cirurgião geral, com diagnóstico de "hérnia umbilical provavelmente encarcerada". 
Relatou aparecimento de abaulamento irredutível e algo doloroso, com saída de secreção, em cicatriz umbilical há aproximadamente 7 meses. Relatou também episódio de melena de grande volume há 5 dias e emagrecimento, não sabendo quantificar a perda. Negava perda do apetite e alterações do trato urinário.

HPP: DM; HAS; cesareana e amigdalectomia.

Ao exame: Descorada. Abdome algo distendido, com tumoração ulcerada em cicatriz umbilical (fig.1), não sendo possível avaliar presença de hérnia; macicez na percussão e sinal do piparote positivo; presença de tumoração endurecida, indolor, palpável e móvel em mesogástrio e hipogástrio; tenso na palpação, mas sem sinais de irritação peritoneal. No toque retal havia abaulamento na parede anterior, com aspecto liso da mucosa retal, sugerindo compressão extrínseca. Toque vaginal de difícil realização que mostrou fundo de saco de Douglas ocupado bilateralmente. Suspeita clínica do Sinal da Irmã Maria José.

Exames laboratoriais de internação: EAS sem alterações; $\mathrm{Ht}=35,9 \% ; \mathrm{Hg}$ $=12,5 \mathrm{~g} / \mathrm{dl}$; leucograma normal; TTPA = $28 \mathrm{seg}$; uréia = $24 \mathrm{mg} / \mathrm{dl}$; creatinina = $0,8 \mathrm{mg} / \mathrm{dl}$; sódio = $142 \mathrm{mEq} / \mathrm{L} ;$ potássio = 3,8 mEq/L; plaquetas $=204.000$ cels $/$ $\mathrm{mm}^{3}$; tempo de protrombina $=13,0$ seg e $82,9 \%$; beta $\mathrm{HCG}=$ não reagente.

Figura 1 - Tumoração vegetante em cicatriz umbilical. 
Tomografia (TC) de tórax = nodulações de contornos irregulares e com calcificações de permeio na gordura pericárdica, não podendo se afastar a possibilidade de implante secundário; linfonodos mediastinais, medindo até 0,5 cm; ausência de derrame pleural; acentuada cifose e sinais de osteoartrose dorsal.

TC abdominal e pélvica: fígado apresentando lesão nodular com focos de calcificação em segmento $\mathrm{V}$, sugestivo de implante secundário; múltiplas formações expansivas com calcificações grosseiras, de limites mal definidos, predominando na cavidade pélvica e assim localizadas (fig.2 e fig. 3): região umbilical $(3,5 \mathrm{~cm} \times 3,3 \mathrm{~cm})$, anexial/parauterina esquerda $(9,6 \mathrm{~cm} \times 9,0 \mathrm{~cm})$, em permeio ao corpo uterino e região anexial/parauterina direita $(9,4 \mathrm{~cm} \times 8,7 \mathrm{~cm})$, adjacente ao reto promovendo efeito compressivo $(4,4 \mathrm{~cm} \times 4,2 \mathrm{~cm})$, e cavidade abdominal em íntimo contato com a parede anterior e perihepática $(3,4 \mathrm{~cm} \times 2,1$ $\mathrm{cm}$ e 1,5 cm x 1,0 cm); linfonodos retroperitoniais calcificados; dilatação ureteral direita por compressão em seu terço médio pela lesão parauterina direita; ausência de líquido livre na pelve. Considerar a possibilidade de lesão primária de origem ovariana com implantes secundários.

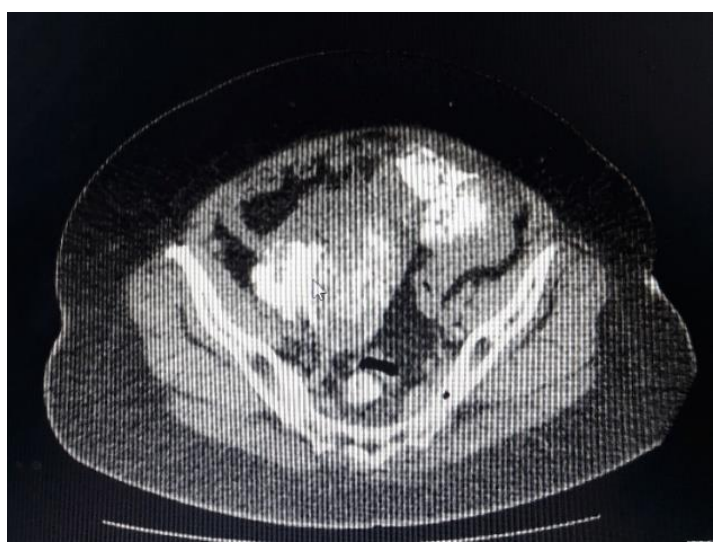

Figura 2 - Múltiplas formações expansivas na com calcificações grosseiras.

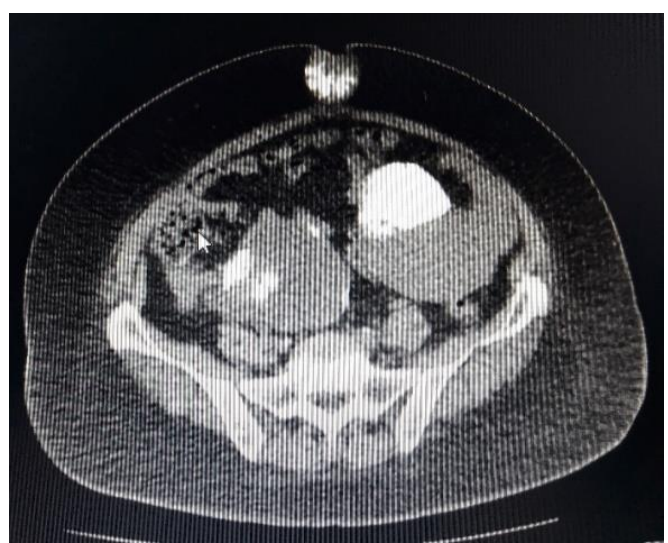

Figura 3 - Formação expansiva cicatriz umbilical. 
Ultrassonografia transvaginal (USG TV): leiomiomas no útero; ovário direito aumentado, com formação cística de paredes finas e componente sólido em sua periferia, medindo 2,1×2,0 cm; ovário esquerdo não visualizado; aumento da ecogenicidade pélvica e moderada quantidade de líquido livre na pelve (implante secundário?).

Colonoscopia revelou: compressão extrínseca de reto.

Endoscopia Digestiva Alta: mostrou hérnia hiatal por deslizamento.

Biópsia incisional da lesão umbilical: adenocarcinoma com padrão micropapilar erodindo a pele; inúmeros corpos psamomatosos; necessário estudo imuno-histoquímico para pesquisa de sítio primário (carcinoma seroso mulleriano? outro?).

Outros exames de laboratório mostraram: CA $19.9=150 \mathrm{U} / \mathrm{ml}$; $\mathrm{CEA}=1$, $0 \mathrm{ng} / \mathrm{ml} ;$ CA $125=12.000,0 \mathrm{U} / \mathrm{ml}$.

Imuno-histoquímica: O estudo imuno-histoquímico revelou positividade para receptor de estrógeno, PAX8 e WT-1, favorecendo o diagnóstico de carcinoma seroso de alto grau; sendo ovário, tuba uterina, útero e epitélio celômico extra-ovariano os sítios primários mais prováveis para este carcinoma.

Foi transferida para o Serviço de Ginecologia, com diagnóstico clínico, laboratorial e de imagem de tumor ovariano avançado, com metástase umbilical.

Foi então encaminhada para Serviço de Oncologia Clínica, onde foi submetida a tratamento quimioterápico.

Após seis meses de tratamento manteve o estado geral e apresentou exame laboratorial que mostrou queda do CA 125 para $>5.000 \mathrm{U} / \mathrm{ml}$. TC de tórax com diminuição das nodulações pericárdicas. TC de abdome e pelve mostrou: diminuição da lesão hepática e da lesão umbilical; discreta dilatação ureteral direita; massa anexial direita de aspecto semelhante e aumento da massa anexial esquerda; redução da massa pélvica adjacente ao reto e colo uterino; redução dos linfonodos retroperitoniais. 
Nódulo da Irmã Maria José - um sinal semiológico de alerta: relato de caso Antônio JLC, Magalhães RR, Pereira KM, Batista ABE, Paula JF, Batista CAM

\section{DISCUSSÃO}

Os nódulos ou lesões na cicatriz umbilical podem ser de origem benigna, maligna ou metastática (PEDRO et al., 2017; PEREIRA et al., 2011; POPARIC et al., 2017; RENDÓN; RIVEROS; HOYOS, 2013).

O achado do Sinal da Irmã Maria José é frequentemente um nódulo endurecido e de crescimento rápido; irregular; podendo ter até $5 \mathrm{~cm}$; fissurado ou ulcerado; com conteúdo seroso, mucinoso, purulento ou sanguinolento; pruriginoso; algo doloroso; de coloração esbranquiçada, violácea ou vermelho acastanhado (FOGAÇA et al., 2003; GIBSON et al., 2018, HUSEIN-ELAHMED et al., 2010; MURINELLO et al, 2010; NIKOLAOU et al., 2013; O'CONNORBYRNE et al., 2020; PEDRO et al., 2017; PEREIRA et al., 2011; POPARIC et al., 2017; RENDÓN; RIVEROS; HOYOS, 2013; VEKARIYA et al., 2020; ZHANG; SANKEY, 2015).

Para confirmação de NIMJ é necessária biópsia incisional ou excisional ou por punção aspirativa, para detectar a origem primária do tumor (FOGAÇA et al., 2003; GATTU et al, 2020; GIBSON et al., 2018; HUSEIN-ELAHMED et al., 2010; MURINELLO et al, 2010; NIKOLAOU et al., 2013; PANARO et al., 2005; PEDRO et al., 2017; PEREIRA et al., 2011; POPARIC et al., 2017; RENDÓN; RIVEROS; HOYOS, 2013; VEKARIYA et al., 2020; ZHANG; SANKEY, 2015).

Histologicamente as tumorações umbilicais, primárias ou metastáticas, são na maioria adenocarcinomas, podendo ainda encontrar carcinomas de células escamosas, indiferenciados e carcinoides (GATTU et al, 2020; HUSEINELAHMED et al., 2010; PANARO et al., 2005; PEDRO et al., 2017; PEREIRA et al., 2011; POPARIC et al., 2017; VEKARIYA et al., 2020); a presença de corpos psamomatosos embora possam ser encontrados na análise de outros carcinomas papilares, são frequentes em carcinoma seroso de ovário e os caracterizam (FOGAÇA et al., 2003; POPARIC et al., 2017).

A imuno-histoquímica é realizada para definição do sítio primário da neoplasia (FOGAÇA et al., 2003; GIBSON et al., 2018; HUSEIN-ELAHMED et 
Nódulo da Irmã Maria José - um sinal semiológico de alerta: relato de caso Antônio JLC, Magalhães RR, Pereira KM, Batista ABE, Paula JF, Batista CAM

al., 2010; MURINELLO et al, 2010, PEREIRA et al., 2011; POPARIC et al., 2017; VEKARIYA et al., 2020). Pode mostrar também se o tumor é receptor de estrogênio e de progesterona, porque a presença desses receptores sugere uma malignidade primária nos órgãos ginecológicos (GIBSON et al., 2018; HUSEINELAHMED et al., 2010).

Uma variação na vascularização e no desenvolvimento embriológico torna o umbigo um alvo fácil para metástases de tumor intra-abdominal, embora esse mecanismo seja pouco conhecido e com várias hipóteses propostas. A forma de aparecimento na região umbilical pode ser por via linfática, hematogênica ou contiguidade (FOGAÇA et al., 2003; GATTU et al, 2020, GIBSON et al., 2018; MURINELLO et al, 2010, PANARO et al., 2005; PEDRO et al., 2017; PEREIRA et al., 2011; POPARIC et al., 2017; RENDÓN; RIVEROS; HOYOS, 2013; VEKARIYA et al., 2020).

Os pacientes podem apresentar simultaneamente ao nódulo sintomas sugestivos de neoplasia, porém, em até $15 \%-45 \%$ dos casos o nódulo é primário e o único sinal da doença, sendo o local primário do tumor desconhecido. Pode ainda significar recidiva (FOGAÇA et al., 2003; GIBSON et al., 2018; MURINELLO et al, 2010; PANARO et al., 2005; PEDRO et al., 2017; RENDÓN; RIVEROS; HOYOS, 2013; VEKARIYA et al., 2020; ZHANG; SANKEY, 2015).

A metástase umbilical é rara (1\%-3\%), sendo o estômago e o cólon no homem e os ovários na mulher os locais primários mais comuns. Pâncreas, vesícula biliar, útero, intestino delgado, mama, entre outros, locais menos comuns (ESPERTO; CANHA, 2012; FOGAÇA et al., 2003; GATTU et al, 2020; GIBSON et al., 2018; HUSEIN-ELAHMED et al., 2010; MURINELLO et al, 2010; NIKOLAOU et al., 2013; PANARO et al., 2005; PEDRO et al., 2017; POPARIC et al., 2017; RENDÓN; RIVEROS; HOYOS, 2013; VEKARIYA et al., 2020; ZHANG; SANKEY, 2015). Pode, mais raramente, ainda ser vista em linfomas (GIBSON et al., 2018; O'CONNOR-BYRNE et al., 2020).

O NIMJ pode ser a primeira e única manifestação clínica da doença. Consequentemente o achado de um nódulo na cicatriz umbilical merece atenção 
Nódulo da Irmã Maria José - um sinal semiológico de alerta: relato de caso Antônio JLC, Magalhães RR, Pereira KM, Batista ABE, Paula JF, Batista CAM

redobrada, avaliação criteriosa e elevada suspeição por parte do médico, para que possa iniciar uma investigação diagnóstica (FOGAÇA et al., 2003; GATTU et al, 2020; GIBSON et al., 2018; HUSEIN-ELAHMED et al., 2010; MURINELLO et al, 2010; NIKOLAOU et al., 2013; PANARO et al., 2005; PEDRO et al., 2017; PEREIRA et al., 2011; VEKARIYA et al., 2020).

Os possíveis diagnósticos diferenciais são: hérnia umbilical redutível ou irredutível, endometriose, cicatriz hipertrófica e queloide, nevo melanocítico, melanoma e granuloma piogênico (FOGAÇA et al., 2003; GATTU et al, 2020; GIBSON et al., 2018; HUSEIN-ELAHMED et al., 2010; MURINELLO et al, 2010; PANARO et al., 2005; PEDRO et al., 2017; RENDÓN; RIVEROS; HOYOS, 2013; VEKARIYA et al., 2020; ZHANG; SANKEY, 2015). Este momento do exame físico é importante e mostra que o profissional de saúde tem que reconheçer esse achado semiológico. No caso aqui reportado a paciente foi encaminhada com diagnóstico de hérnia umbilical complicada.

Exames complementares, como colonoscopia, endoscopia digestiva alta, TC abdominal, ressonância nuclear magnética, USG abdominal, USG endoscópica e USG TV, são necessários e utilizados na investigação diagnóstica do sítio primário (ESPERTO; CANHA, 2012; FOGAÇA et al., 2003; GATTU et al, 2020; GIBSON et al., 2018; HUSEIN-ELAHMED et al., 2010; MURINELLO et al, 2010; NIKOLAOU et al., 2013; PANARO et al., 2005; PEDRO et al., 2017; PEREIRA et al., 2011; POPARIC et al., 2017; RENDÓN; RIVEROS; HOYOS, 2013; VEKARIYA et al., 2020; ZHANG; SANKEY, 2015).

Marcadores tumorais, como CA 19.9, CA 125, CEA, também são usados na avaliação do paciente para a identificação do sítio primário (FOGAÇA et al., 2003; GIBSON et al., 2018; PEDRO et al., 2017; PEREIRA et al., 2011; POPARIC et al., 2017; VEKARIYA et al., 2020).

$\mathrm{Na}$ paciente em questão, a hipótese de neoplasia ovariana foi aventada pela presença do sinal semiológico e pelos achados de imagens na TC abdominal. Foi então reforçada e confirmada pela positividade do CA 125, CA 19.9 e a imuno-histoquímica, conforme mostra a literatura, que afirma que 0 
aumento do CA 125 pode ser observada em 90\% dos pacientes com adenocarcinoma de ovário, principalmente naqueles do tipo seroso, tendo este tipo de tumor ovariano existe importante correlação entre a positividade do teste imuno-histoquímico e os níveis séricos elevados de CA-125 (FOGAÇA et al., 2003; NIKOLAOU et al., 2013; PEREIRA et al., 2011; POPARIC et al., 2017).

O tratamento varia de cirurgia agressiva com terapia adjuvante, dependendo do tipo de tumor, da sua origem primária e também do estado clínico do paciente ao tratamento paliativo (quimioterapia, radioterapia ou ambos). Em alguns casos nenhum tratamento é possível de ser realizado devido ao falecimento precoce do paciente (FOGAÇA et al., 2003; GATTU et al, 2020; GIBSON et al., 2018, HUSEIN-ELAHMED et al., 2010; MURINELLO et al, 2010; NIKOLAOU et al., 2013; PEDRO et al., 2017; PEREIRA et al., 2011; POPARIC et al., 2017; RENDÓN; RIVEROS; HOYOS, 2013; VEKARIYA et al., 2020). No presente caso decidiu-se pelo tratamento paliativo em função do estadiamento avançado da doença.

O NIMJ indica doença avançada e está associado a mau prognóstico, com uma sobrevida média de 11 meses. Porém o que define o prognóstico é principalmente a origem primária da neoplasia (ESPERTO; CANHA, 2012; FOGAÇA et al., 2003; GATTU et al, 2020; GIBSON et al., 2018; HUSEINELAHMED et al., 2010; MURINELLO et al, 2010; NIKOLAOU et al., 2013; PANARO et al., 2005; PEDRO et al., 2017; PEREIRA et al., 2011; POPARIC et al., 2017; RENDÓN; RIVEROS; HOYOS, 2013; VEKARIYA et al., 2020; ZHANG; SANKEY, 2015).

\section{CONCLUSÃO}

O sinal semiológico da Irmã Maria José pode ser a primeira e única manifestação clínica de uma neoplasia. Portanto, perceber uma lesão na cicatriz umbilical durante o exame físico e conhecer os seus diagnósticos diferenciais é 
de suma importância, pois permite iniciar uma investigação diagnóstica e propor uma medida terapêutica adequada ao caso.

\section{AGRADECIMENTOS}

Nossos agradecimentos a Sra. Rosalynn Leite Teixeira, bibliotecária do Curso de Medicina da UNIFAA, pela ajuda no levantamento bibliográfico do trabalho.

\section{REFERÊNCIAS}

ESPERTO, H.; CANHA, C.; MEIRA, E. Nódulo da Irmã Maria José. Acta Med Port, v. 25(4), p. 258-258, 2012.

FOGAÇA, H. S. et al. Nódulo Umbilical Metastático (Nódulo da Irmã Maria José): Um Sinal de Alerta para Tumoração Maligna Intra-abdominal - Relato de Caso. RBGO, v. 25(6), p. 449-452, 2003.

GATTU, R. et al. Sister Mary Joseph nodule: an often overlooked or misdiagnosed entity on imaging. Clinical Imaging, v. 60, p. 177-179, 2020.

GIBSON, J. A. G. et al. Sister Mary Joseph nodule: a diagnostic challenge. BMJ Case Rep, 2018. doi:10.1136/bcr-2017-223674.

HUSEIN-ELAHMED, $\mathrm{H}$. et al. Sister Mary Joseph's nodule as a metastasis of ovarian adenocarcinoma. International Journal of Dermatology, v. 49, p. 1045-1046, 2010.

MURINELLO, A. et al. Sister Mary Joseph's nodule. J Port Gastrenterol, v. 17, p. 266-270, 2010.

NIKOLAOU, M. et al. Sister Mary Joseph's nodule in advanced ovarian câncer. J Obstet Gynaeco, v. 33(2) p. 214-215, 2013.

O'CONNOR-BYRNE, N. et al. Sister Mary Joseph nodule in mantle cell lymphoma. Lancet Oncol, v. 21: e337, 2020. 
PANARO, F. et al. Sister Joseph's nodule in a liver transplant recipient: Case report and mini-review of literature. World J Surg Onc 3, 4 (2005). https://doi.org/10.1186/1477-7819-3-4.

PEDRO, B. et al. Nódulo de Irmã Mary-Joseph - A primazia da clínica. Rev Clin Hosp Prof Dr Fernando Fonseca, v. 5 (1/2), p. 41-42, 2017.

PEREIRA, W. A. et al. Sister Mary Joseph's nodule: a sign of internal malignancy. An Bras Dermatol., v. 86(4Supl1), p. 118-120, 2011.

POPARIC, S. et al. Sister Mary Joseph Nodule in an Ovary Adenocarcinoma. Med Arch, v. 71(2), p. 154-157, 2017.

RENDÓN, L. M. G.; RIVEROS, M.; HOYOS, A. V. Nódulo de la Hermana María José: Un reporte de caso. MEDICINA U.P.B., v. 32(1), p. 92-95, 2013.

VEKARIYA, P. et al. Sister Mary Joseph Nodule as an Initial Presentation of Pancreatic Adenocarcinoma. ACG Case Rep J., v.7, e00453, 2020.

ZHANG, L; SANKEY, C. The Sister Mary Joseph Nodule. J Gen Intern Med, v. 30(5), p. 689-690, 2015. 